\title{
Proposed Approach for Enhancement of QoE to Support e-Society*
}

\author{
Sufyan T. Faraj Al-Janabi \\ ${ }^{2}$ College of Science and Technology \\ UHD, Sulaimani, KRG-Iraq \\ ${ }^{1}$ College of Computer Science and IT \\ University of Anbar, Ramadi, Iraq \\ saljanabi@fulbrightmail.org
}

\author{
Dana Kareem Hama Ghareeb \\ Dept. of Computer Science \\ School of Science \\ University of Sulaimani \\ Sulaimani, KRG- Iraq \\ dancok85@gmail.com
}

\begin{abstract}
In recent years, multimedia services and social media applications have become extremely popular as they have been accessed by laptop computers and mobile devices. These can be considered as basic communications functionalities required for building e-society. The heterogeneity of hardware capabilities and network environments result in big challenges for service providers in always providing users with a satisfying experience. The meaning of Quality of Experience (QoE) reflects the degree of a user's subjective satisfaction, which can be different from the Quality of Service $(\mathrm{QoS})$ concept as QoS is solely related to objective system performance metrics, such as the bandwidth, delay, and loss rate. In this work, we propose a general approach for improving QoE through QoS such as to support various e-society applications and services. Various models and parameters related to this issue are reviewed. The proposal represents a framework that considers different aspects such as classification, marking, routing, and queuing to achieve better controlling of relative QoS parameters.
\end{abstract}

Keywords-Quality of service; Quality of Experience; Mean opinion score, DiffServ- Differentiated Services; IntServIntegrated Services

\section{INTRODUCTION}

During last decade social media applications have been the most interested applications to the end-users. These applications are the biggest challenge to network providers because of the sensitivity of these real-time applications influenced by Quality of services (QoS) parameters. Undoubtedly, one of the essential topics in the area of data networking is QoS. The term QoS is used to explain network performance such as resource availability, delivery, and capacity. But the Quality of Experience (QoE) is defined as the measure of user satisfaction and performance based on subjective and objective psychological measures of using a service or product [1].

The International Telecommunication Union (ITU-T) defines QoE as the "the overall acceptability of an application or service, as perceived subjectively by the end-user" QoE is different from network QoS indicators (e.g., bandwidth, loss rate, jitter), which are not adequate to get a precise idea about the visual quality of a received video sequence.
QoE instead focuses on the overall experience of the end user. It depends on the overall system behavior, starting from the source of the services up to the end user, including the content itself and the network performance [2], [3].

To accomplish the ultimate goal of user satisfaction, QoS solutions are necessary. But, they are not sufficient. As QoS consider quality as a pure technical, we need to deploy QoE solutions in order to consider the quality from a user's point of view. Using QoS techniques in relation to the QoE solutions would enable us to present an adequate level of quality for the users. We can collect data about provider's user's experiences, analyze them, and then calculate the value of participating QoS factors in relation to QoE [4]. So we need to use a quantitative relationship between QoS and QoE in respect to the correlation between QoS and QoE, and then develop a framework to improve QoE through the QoS.

The remaining of this paper is organized as follows: In Section 2, we present a survey on some important related literature. Sections 3 and 4 explain various aspects of QoS and QoE in detail, respectively. Then, Section 5 gives the relation between QoS and QoE. In Section 6, our proposed general framework for the enhancement of QoE is introduced. Next, Section 7 represents a case study for implementing the proposed framework on internet service provider (ISP) network. Finally, Section 8 concludes the paper.

\section{RELATED WORK}

The QoS of a given system is expressed as a set of (parameter-value) pairs, sometimes called QoS requirements. We consider each parameter as a typed variable whose values can range over a given set. Different applications on the same distributed system can have different subsets of relevant QoS parameters or requirements as was presented by Andreas Vogel et al in [5].

For the VoIP applications as presented by Xiuzhong Chen et al, QoS has different requirements including packet loss, delay, and jitter. Current H.323 and SIP frameworks allow the users and the network to reach an appropriate service agreement such that the network can allocate resources to enable QoS guarantees [6]. 
According to Zapater and Bressan, the QoS gives some high-level directions ranging from design to management of IPTV service delivery infrastructure. Such directions aim to help service providers to meet customers' QoE requirements and to transition IPTV from small scale deployments to the mass market successfully [7].

Despite the fact that different architectures (Including Integrated Services, Differentiated Services, MPLS, and Traffic Engineering) have already been proposed for achieving QoS, providing end to end QoS is still a real networking challenging particularly for supporting multimedia services. An integrated framework had been considered where there was an interaction between QoSaware video application and DiffServ network. The Triage algorithm was used to facilitate content-aware service differentiation based on relative individual QoS packet requirements. This proposal had taken the advantage of the unequal importance of video packets in order to preserve information that is most important to spatial/temporal quality [8].

Möller et al worked to used QoS to improve multilayer Networks-on-Chip (NoC) that allows several data transfers to occur in parallel and are indeed the communication infrastructure of future hundred-cores Systems-on-Chip (SoCs) [9].

Furthermore, other frameworks had been presented to enhance QoE concept such as the flexible QoE framework for video streaming services. The new service evaluations are QoE metrics to achieve high-performance network quality [10]. In the respect, there are various types of performance that can be considered such as Network Performance, Network Performance Overall, QoE, and end-to-end QoS.

The effective QoS measurement of Web Browsing Services in $3 \mathrm{G}$ networks was presented by Haryadi and Nusantara in [11]. Also, it was found that the QoS routing in next-generation high-speed networks is satisfying the QoS requirements for every admitted connection, and achieving global efficiency in resource utilization, to support a wide range of communication-intensive realtime multimedia applications [5]. Even more, the QoS in WiMaX "Worldwide Interoperability for Microwave Access" was found to have different QoS requirements to support different applications for better QoS as mentioned by Malhotra and Priyanka in [12].

QoE management framework, called "In-service feedback QoE framework", was proposed where end users give feedback immediately whenever service dissatisfaction occurs. This user-triggering scheme initiates an investigation to find out which factors dominantly deteriorated the quality. Gathered feedback information from distributed end users, from servers can be analyzed collectively to find out the reason and location of faults [13].
The main aim of our work is to propose a general framework to improve QoE through QoS concept and parameters. This proposal is assumed to be used by ISP to successfully achieve users' satisfaction for various social multimedia services. This can be considered as a basic step towards building the e-society.

\section{QUALITY OF SERVICE}

The term of QoS includes a wide range of technology, parameters, architecture, and protocols. Service providers complete end-to-end QoS by ensuring that all network elements work effectively and control all traffic over the network. In another word, QoS provisions the set of qualitative and quantitative characteristics of a distributed network system to reach the required functionality of an application [5].

With this fast evolution of high-speed networks and networked services supplying differentiated services in the network to the user, QoS became more and more essential [14]. QoS is based on objective system performance metrics such as the loss rate, bandwidth and delay [15]. This means providing the network that is transparent to its users [16]. Usage of social media applications and multimedia application is increasing day by day in the human social life, medical, military and businesses. The usability or the success of continuous multimedia application depends largely on the QoS [17].

To implement QoS, the following steps must be done:

- First, identifying the traffic and its requirements.

- The second dividing traffic into classes like class models voice, call signaling, critical data, video, etc.

- Third, defining QoS policies for each class to prioritize traffic over other traffic.

\section{A. Models of QoS}

There are several main models of QoS that are reviewed below (Table 1 represents a comparison of these three models):

- Best-effort: Internet initially used best-effort as a default mode for packet delivery services. Best-effort is the default mode for all traffic. In this mode, there is no differentiation among types of traffic. Benefits of the Best-effort model are high scalability and no special mechanisms are required. The drawbacks of best-effort are no service guarantees and no service differentiation. The basic queuing during congestion in the best-effort mode is the first-in first-out (FIFO) packet delivery strategy. The Internet generally uses "Best Effort" approach which is associated with IPv4, in which content of the packet is not sensitive to the real-time data flow. As demand exceed capacity, service degrades, thereby causing jitters, packet loss and delays; which a big hitch to real-time applications [18], [19]. 
- IntServ: IntServ is a model that ensures guaranteed delivery and predictable behavior of the network in end to end fashion. In this model, the Resource Reservation protocol (RSVP) is usually considered as a signaling protocol. That is requested for enough bandwidth to send packets. Intserv used in special cases voice and video because it is more sensitive. Indeed, this model requires the deployment of intelligent queuing mechanisms to provide resource reservation. The benefits of the IntServ model are explicit resource admission control and signaling of dynamic port numbers (for example, H323). The main drawback is that it is not good for global Internet [19].

- DiffServ: DiffServ is different from IntServ; even with no enough bandwidth it is possible to send packets through the path. DiffServ works according to the need of the traffic that can be classified. In this model, it is possible to treat some traffic better than the rest. But, the DiffServ does not have hard and fast guarantee. Thus, proper network engineering is necessary in order that DiffServ provides handling for a wide class of applications [19].

\section{B. QoS Categories and Mechanisms}

Many mechanisms might be considered to achieve good QoS for any services, such as:

- Classification: It is the core of QoS. It is the ability to identify different traffic types and prioritize one to other, usually by using two tools Access control list (ACL) and network base application recognition (NBAR).

- Marking: It is the work to coloring packets which gives the priority to be identified in other routers. Marking tools include CoS (Class of Services) for switches and ToS (Type of Services) which is used in layer three by routers.

- Policing: It is connected to the type of QoS. For example, in QoS tools that take weight bandwidth, any packets reached the limited weight must be dropped.

- Shaping: Allows dealing with interfaces if the speed of the router is faster than all network devices, to keep balancing. Shaping works to queue these packets and send them later.

- Congestion Avoidance: It usually uses two techniques; Random Early Detection (RED) and Weight Random Early Detection (WRED). Here, if a user is sending packets with TCP, and the detector notices that this user is making congestion in the network, it drops the packets randomly to avoid the congestion.

- Queuing: It can be the most powerful category of QoS. Many queues are used to achieve a good QoS like LLQ, CBWFQ, PQ, Tree queuing, etc.
Table I. QoS basic models.

\begin{tabular}{|l|l|l|}
\hline \multicolumn{1}{|c|}{ Best-Effort } & \multicolumn{1}{|c|}{ IntServ } & \multicolumn{1}{c|}{ Diffserv } \\
\hline $\begin{array}{l}\text { No Q0S is applied to } \\
\text { packets. best-effort is the } \\
\text { default mode for all traffic }\end{array}$ & $\begin{array}{l}\text { Application signal to the } \\
\text { network that they require } \\
\text { certain Q0S parameters }\end{array}$ & $\begin{array}{l}\text { The network recognizes } \\
\text { classes that require Q0S }\end{array}$ \\
\hline $\begin{array}{l}\text { No service guarantees } \\
\text { No service differentiation }\end{array}$ & $\begin{array}{l}\text { Used (RSVP) protocol as } \\
\text { signaling protocol and } \\
\text { reserved all the } \\
\text { bandwidth until finished } \\
\text { connection and block } \\
\text { other traffics. } \\
\text { Service guarantees }\end{array}$ & $\begin{array}{l}\text { The packet-handling rule } \\
\text { is termed as Per-Hop } \\
\text { Behavior (PHB).Here if } \\
\text { traffic has high priority, } \\
\text { It's classify and remark to } \\
\text { pass through all networks } \\
\text { without straggle. }\end{array}$ \\
\hline $\begin{array}{l}\text { Because it's default mode } \\
\text { for all traffic don't have } \\
\text { any sensitivity with } \\
\text { traffics. Internet was } \\
\text { initially based on best } \\
\text { effort packet delivery and } \\
\text { don't have special } \\
\text { prioritize. }\end{array}$ & $\begin{array}{l}\text { End-to-End streams are } \\
\text { not established if the } \\
\text { required Q0S are not } \\
\text { available }\end{array}$ & $\begin{array}{l}\text { Even without enough } \\
\text { bandwidth the services } \\
\text { work fairly. }\end{array}$ \\
\hline $\begin{array}{l}\text { Used technique FiFS } \\
\text { queuing to deal with } \\
\text { traffic }\end{array}$ & $\begin{array}{l}\text { Used PQ to deal with } \\
\text { traffic }\end{array}$ & $\begin{array}{l}\text { Used CBIVQ, Tree } \\
\text { Queue and LLQ to deal } \\
\text { with traffic }\end{array}$ \\
\hline $\begin{array}{l}\text { No special mechanisms } \\
\text { required. But highly } \\
\text { scalable. }\end{array}$ & $\begin{array}{l}\text { Used in special case } \\
\text { because of sensitivity of } \\
\text { this model have many } \\
\text { requirements cause high } \\
\text { cost challenge for } \\
\text { implements used in } \\
\text { private office not in global } \\
\text { internet. }\end{array}$ & $\begin{array}{l}\text { It's different from other } \\
\text { two models; According to } \\
\text { need the traffic } \\
\text { reprioritize. Even with not } \\
\text { enough bandwidth the } \\
\text { packets can pass through } \\
\text { the path. }\end{array}$ \\
\hline $\begin{array}{l}\text { There is no differentiation } \\
\text { among types of traffic. }\end{array}$ & $\begin{array}{l}\text { The problem in Intserv is } \\
\text { reserving and blocking } \\
\text { only particular traffic can } \\
\text { use the bandwidth not all } \\
\text { traffic. }\end{array}$ & $\begin{array}{l}\text { the traffic is treated } \\
\text { according to its respective } \\
\text { classes }\end{array}$ \\
\hline
\end{tabular}

\section{QoS Layering}

Application-dependent parameters and applicationindependent parameters are two main subsets of QoS parameters [20], [21]. It is possible to view them based on a general architecture of three layers: application, system, and network layers (Table 2 summarizes this layering concept) [20]:

1. System layer: In this layer, we can further subdivide system parameters into: network and operating system parameters, and device parameters.

2. Application layer: The parameters in this layer usually represent the requirements for application services. These parameters can be described in terms of both media quality and media relations. The parameters of Timeliness, Accuracy, and Precision (TAP) can together be considered as a good criterion for QoS and can be specified in either quantitative or qualitative terms.

3. Network layer: The parameters of the network layer can be considered in terms of both network load (Which is related to ongoing traffic requirements such as packet inter-arrival time) and network performance (Which specifies the requirements that must be guaranteed such as bandwidth and delay). In the next subsection, we present QoS parameters. 
Table II. QoS layers [20].

\begin{tabular}{|c|c|}
\hline QoS Layer & QoS Parameters \\
\hline Application & $\begin{array}{c}\text { Frame Rate, Frame Size and Resolution, Response Time } \\
\text { Throughput, Security, Price and Convenience }\end{array}$ \\
\hline System & $\begin{array}{c}\text { Buffer Size, Process Priority, Scheduling Policy } \\
\text { Caching Policy, Time Quantum }\end{array}$ \\
\hline Network & $\begin{array}{c}\text { Bandwidth, Throughput, Bit Error Rate, End-to-End Delay } \\
\text { Delay Jitter, Peak Duration }\end{array}$ \\
\hline
\end{tabular}

\section{QoS Parameters}

The QoS parameters must be guaranteed if the network capacity is limited [21]. The most important QoS parameters are:

- Delay: End-to-end transit delay is the elapsed time for a packet to be passed from the sender through the network to the receiver [17]. In other research studies, delay defined as the time taken to establish a particular service from the initial user request and the time to receive specific information once the service is established. Delay can make the system unusable and unresponsive especially for interactive or real-time applications. Delay is a significant QoS challenge for applications such as Torrents, Viber, Tango and Facebook. Finally, the delay can be both fixed and variable [22].

- Jitter: The variation in end-to-end transit delay is called jitter (delay variation) [17]. Jitter can also be defined as the measure of delay variation between repeated packets for a given traffic flow [22]. High levels of jitter are unacceptable in situations where the application is real-time. The strong interconnection between the end-to-end delay and the jitter should be noted.

- Bandwidth: The maximal data transfer rate that can be sustained between two end points of the network is defined as the bandwidth of the network link [17]. In fact, bandwidth is possibly the second most significant parameter that has the real impact on QoS [22]. It should be noted that the bandwidth is not only limited by the physical infrastructure of the traffic path within the transit networks, which provides an upper bound to the available bandwidth but is also limited by the number of other flows sharing common resources on this end-to-end path.

- Loss: The physical errors introduced by transmission media cause packet loss. Wireless channels (such as satellite and mobile networks) usually have high Bit Error Rate (BER) compare to wired channels (e.g., fiber optics). Protocols like TCP protect against packet loss by using packet retransmission strategies [22].

\section{QUALITY OF EXPERIENCE}

The customer satisfaction degree of a service called Quality of Experience (QoE). The notion of QoE has been raised during the last decade with the meaning of involving the subjective understand of end-users into the evaluation of telecommunication services quality [23]. In addition, QoE is the overall suitability of an application or service, as perceived subjectively by the end users [24]. In general over the years, network operators have estimated network performance based on QoS parameters. Hence, it is essential to understand the relationships between useroriented QoE and network-oriented QoS parameters [23].

The QoE as a collection of QoS and human userrelated metrics will be the key success factor for current and future services provider. Network operators want to understand how to minimize network churn by providing better services to the users. On the other hand, network engineers require the knowledge about underlying network conditions affecting users QoE for user-centric network optimization [25]. It is important for the service provider to understand the quantitative relationship between QoE and these technical parameters in order to manage the user perceived quality [26].

One of the biggest challenges in this respect is to deal with various QoE metrics considering the effect of multiple confusing factors. These include technical, social, psychological, and physiological factors. One important characteristic user-related measure is the mean opinion score (MOS). This can be determined from subjective ratings by real users or predicted from objective measurements of properties of the delivered goods such as audio, video, or files [27], [28]. Figure 1 depicts the main assessment methods for QoE.

Fig. 1. QoE Assessment Methods [27].

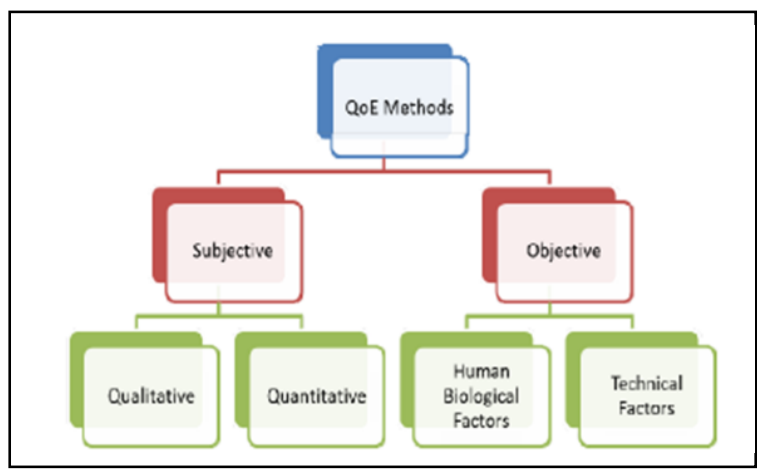

The first category of assessment methods is subjective assessment. In these methods, interviews and surveys are usually used. Then, statistical sampling of users is done in order to analyze their needs and perceptions. The main task here is to understand human subjectivity and transform it into meaningful data with very high degree of accuracy [29]. The subjective methods can further be subdivided into qualitative and quantitative techniques. Qualitative data consists of words and comments. Thus, qualitative techniques deal with the human verbal behavior. One important framework in this direction is the CCA (catalog, categorize, analyze) framework.

On the other hand, quantitative factors consist of numbers and statistics. Hence, quantitative techniques have to combine the survey with rating scales in order to produce quantitative data. Various guidelines for subjective studies have been issued by the International 
Telecommunications Union (ITU). These include P.910 Recommendation for video quality, G.1030 Recommendation for web traffic quality, and P.800 Recommendation for speech quality [27].

The second category consists of the objective QoE assessment methods. These can also be divided into QoS technology centric and secondly human physiological cognitive-based techniques. For example, objective methods for the evaluation of picture quality include Full Reference (FR), No Reference (NR), or Reduced Reference (RR) methods [28].

Peak Signal to Noise Ratio (PSNR) is one well known traditional objective measure used to assess video quality. However, PSNR has its limitations. Thus, other measures for video quality had been proposed such as Moving Pictures Quality Parameter (MPQM). Subjective video quality measurement cannot provide real-time and in-service quality monitoring for real-time video applications. So the application of the method is limited [29].

For subjective evaluation methods, opinion rating (Mean Opinion Score- MOS) based on customer's satisfaction was proposed to assess the perceptual QoS. It is specified in ITU-T recommendations E.800 initially. On another hand, several objective quality assessed methods had been proposed in ITU-T, such as P.861 PSQM (Perceptual Speech Quality Measure), P.862 PESQ (Perceptual Evaluation of Speech Quality) and G.107 EModel.

MOS basic definition of can be found in ITU-T Rec. P.10 as: "the mean of opinion scores, i.e., of the values on a predefined scale that subjects assign to their opinion of the performance of the telephone transmission system used either for conversation or for listening to spoken material." Five-point scale for MOS was defined by ITUT Recs. P.800 and P.800.1. Different estimate methods are needed for accurate MOS measurement. MOS textual descriptions can be classified into quality oriented or impairment oriented (See Table 3). QoE evaluation of each service or application requires the choice of suitable metrics [30], [31].

\section{THE RELATIONSHIP BETWEEN QOS AND QOE}

If we use QoS techniques in relation to the QoE solutions, then it would be possible to present an adequate level of quality for the users. So we need to use a quantitative relationship between $\mathrm{QoS}$ and QoE in respect to the correlation between QoS and QoE. We can collect data about provider's users' experiences, analyze them, and then calculate the value of participating QoS factors for related QoE parameters. Then, we can try to enhance the QoS solutions according to QoE requirements [4].

A large number of studies connecting between QoS and QoE, still often focusing only on overall user perceived quality (often in terms of MOS). The relationship of different dimensions of QoS and QoE, in particular for classifying interactive multimedia environments, identifying the degree to which different QoS factors impact different QoE dimensions [32]. In general, it is believed that the correlation relationship between QoE and QoS can be described by either logarithmic or exponential equations. However, there is no general agreement on which type of equation has more accurate description capability.

Table III. Different types of discrete metrics [31].

\begin{tabular}{|c|c|c|}
\hline \multicolumn{3}{|c|}{ (a) Absolute metrics } \\
\hline Mos & Quality & Impairment \\
\hline 5 & Excellent & Imperceptible \\
\hline 4 & Good & Perceptible \\
\hline 3 & Fair & Slightly annoying \\
\hline 2 & Poor & Annoying \\
\hline 1 & Bad & Very annoying \\
\hline \multicolumn{3}{|c|}{ (b) Comparative metrics } \\
\hline Score & \multicolumn{2}{|c|}{ Description } \\
\hline 3 & \multicolumn{2}{|c|}{ Much Better } \\
\hline 2 & \multicolumn{2}{|l|}{ Better } \\
\hline 1 & \multicolumn{2}{|c|}{ Slightly Better } \\
\hline o & \multicolumn{2}{|c|}{ About the Same } \\
\hline-1 & \multicolumn{2}{|c|}{ Slightly Worse } \\
\hline-2 & \multicolumn{2}{|l|}{ Worse } \\
\hline-3 & \multicolumn{2}{|c|}{ Much Worse } \\
\hline
\end{tabular}

The psychological stimulus-centric Weber-Fechner Law (WFL) has been used to propose a logarithmic relationship between QoE and QoS. The obtained logarithmic QoE-QoS relationship can be described as [33]:

$$
Q o E=k \cdot \ln (Q o S)
$$

where $\mathrm{k}$ is a constant that is determined according to the experiment environment. Other people have adopted a perception-centric approach based on the IQX-hypothesis. This hypothesis claims that changes in QoE are related to user quality perception levels. This approach results in the following exponential relationship for describing QoEQoS [33]:

$$
Q o E=\alpha \cdot e^{-\beta \cdot Q o S}
$$

\section{A GENERAL PROPOSED FRAMEWORK}

The general proposed framework has two main parts; QoS part and QoE part. In addition, the QoS part is divided into main subparts; data plane and control plane (See Figure 2). We propose to use classification and queuing techniques. The classification identifies packets and marks them by using ACL (Access control list) via IP address or switch port and application type via NBAR. The queuing deals with high-performance memory buffer in the router that holds data to be processed. Queuing works with base priority queue and CBQ (class base queue). In a priority queue, the traffic with high priority is going first. In CBQ, the traffic is kept wait in the queue and unimportant traffic is dropped. Another important QoS issue is to reduce the network congestion by using RED and WRED tools. 


\section{A. QoS Mechanisms in Data Plane}

It is possible to map data plane mechanisms into the corresponding TCP/IP layers. The network layer QoS mechanisms are used to implement the control functions of packet forwarding by controlling the relative per-hop behavior (PHB). These mechanisms include packet classification, buffer management, scheduling, shaping, and policing

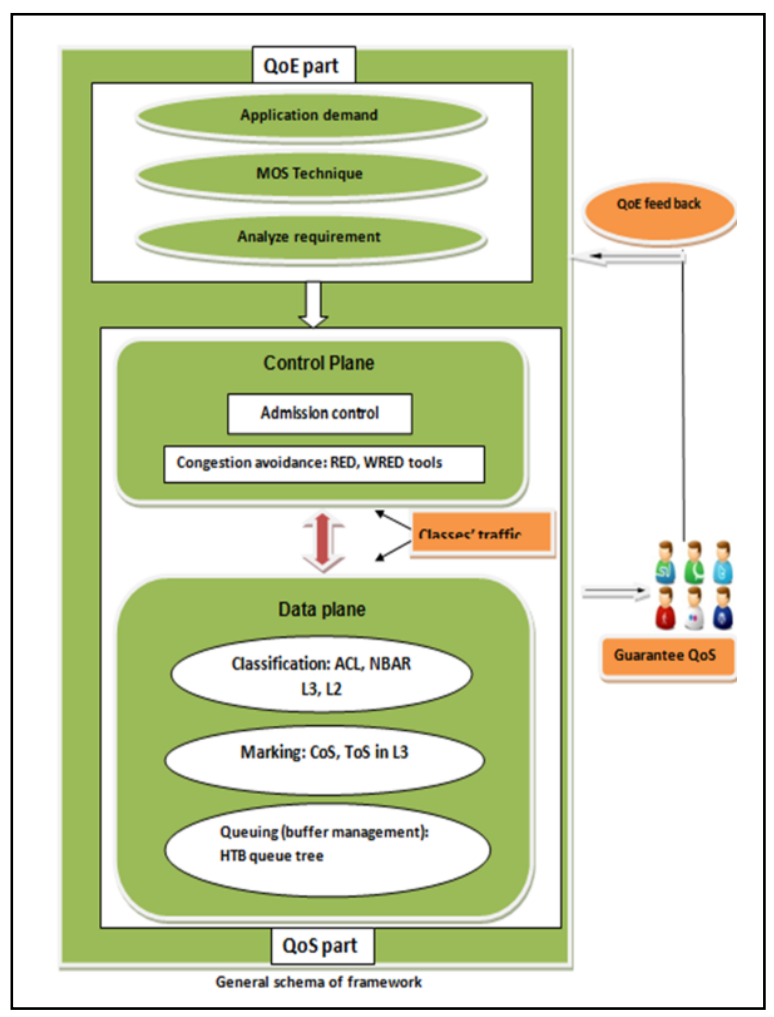

Fig. 2. The General Proposed Framework.

In this plane, our proposal is based on DiffServ that presents a framework of a service architecture within which both enterprise and ISPs can offer differentiated services to their customers on the basis of performance. DiffServ architecture has been approved by IETF in RFC 2475. The so-called Differentiated Service Code Point (DSCP) is used as a code point to select the PHB. At the network node output, the PHB delivers the DiffServ to packets including policing, shaping, possible remarking of DSCP, queuing treatment and scheduling.

There are two major services in DiffServ which are Better Best Effort and Virtual Leased lines. One important advantage of DiffServ is that it works with various networking protocols. Another important advantage is that DiffServ facilitates the scaling of the Internet at the physical speed (i.e. Gbps/link) [34].

Usually different techniques for QoS are required in different networking environments. This is a direct consequence of the necessary trade-offs among QoS service, operation complexity, and cost of implementation. Buffer management and scheduling are needed to ensure that service quality. In buffer management, packets are usually dropped when the queue is (almost) full. The scheduling policy is used to maintain control on queuing delay and bandwidth sharing. Most important examples of scheduling techniques are First Come First Serve (FCFS), Static Priority (SP), and Earliest Deadline First (EDF). By default, all routers support FCFS in the best-effort model while many new devices also support (CBWFQ) Tree Queue.

\section{B. QoS Mechanisms in Control Plane}

Multimedia applications are delay-sensitive and lossinsensitive. It was shown that the primary parameter affecting the quality of voice on the Internet is the end-toend delay. End-to-end delay definition constitutes of the sum of propagation, processing, serialization, and queuing delays.

In the control plane, QoS can be achieved using mechanisms like traffic engineering and admission control. Admission control means management of packets and trying to give them a good QoS, but with a limited network resource, it will be difficult to offer all QoS requirements for all applications. Unfortunately, many networks currently still do not offer admission control. This result in significant QoS reduction because such networks allow new traffic to keep entering even beyond the network capacity. To prevent this, admission control mechanisms should be at the place. The general architecture of admission control unit is shown in Figure 3.

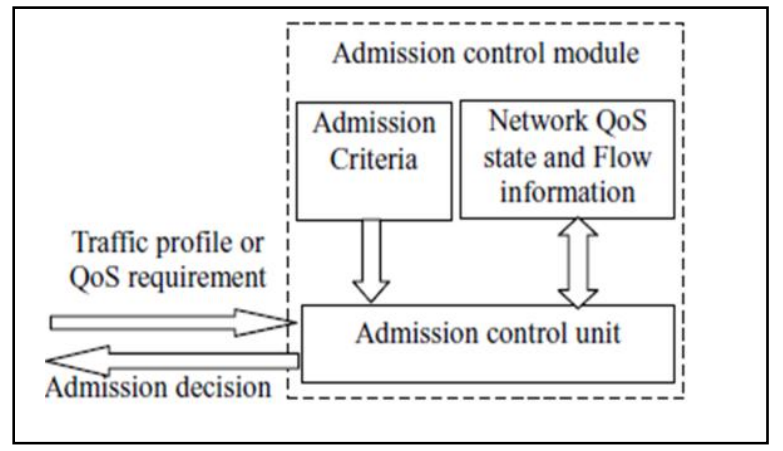

Fig. 3. Components of admission control [6]

\section{The QoE Part}

In our model, we are proposing measuring the end user experience via adding a perceptual upper-layer, which is responsible for the QoE metric. In the Application Layer, the QoS is driven by the human perception of audio and video. The perception is based on three main characteristics: spatial perception, temporal perception (for video) and acoustic band-pass (for audio). Applications such as Teleconferencing, VoIP, distance learning, etc. make use of these characteristics. The network QoS solutions provide different relative levels of QoS from highest to lowest quality. These differential solutions can complement each other when implemented as part of QoS Architecture. The QoS mechanism to be used is driven by the application, network topology, and the policy to be applied. 
In the QoE part application metrics, the MOS is used to make informed management decisions. The main difference of our approach compared to other existing solutions is that we use information from different layers, and combine them at both edges of the broadband network to provide a complete resource management platform. We predominately evaluate our proposal in our tests by comparing the perceived user experience, i.e., QoE in order to refine various framework parameters. QoS is an objective measure of the service delivered while QoE is a subjective quality technique which uses CCA (Catalog Categorize Analyze) framework and quantitative metric. Thus, network providers that provide good QoE have a significant competitive advantage over those who are only interested in QoS measures.

\section{CASE STUdY}

We have proposed a general framework to support QoE features through QoS mechanisms. The proposal provides resource assurance with Diffserv including congestion control, admission control, traffic classification, traffic marking, and traffic queuing. In this section, we report on our ongoing work to apply and test the protocol on a sample ISP provider network. This case study will enable us to better refine our proposal in an effective manner. In order to maximize the end user experience, we collect and analyze data from the selected ISP network such as to enhance QoE through controlling the QoS model and metrics.

In the QoE part, we use feedback from end users about the services and analyze these results. Subjective mechanisms like mean opinion score (MOS) are to be applied. Feedback can be obtained online from simple survey documents that can be filled by users for some extra bonus from the ISP. The analysis is to be done based on network QoS parameters, such as bandwidth, delay, and packet loss. The obtained information is used to predict the quality of many social media applications like Facebook, Viber, Tango, etc. Finally, QoS parameters are refined based on these findings.

We expect this approach to be helpful for ISPs to better understand how to control and manage their networks' various parameters and resources to offer a satisfactory level of QoE for future e-society services. This case study considers real ISP network in KRG. The network contains two main servers (Mikrotik server model core 1063), several sections, and provides internet services for about 3000 end users. It works with fiber optic link (Cor2, SFE), Microwave link (Extend Air G2 rc070207 GHz), and many Mikrotik dishes (Net Metal/1600 mW, RB 12/1000 mW, R5000-Lmn). Thus, there is a big challenge of QoE requirements with increasing user demands for social networking.

For the QoS part, the following two mechanisms will be considered in the control plane:

1. Admission control: The admission control function is more challenging in heterogeneous networks. The common parameters used for admission control are summit bandwidth requirement and the average rate.
2. Congestion control: In the QoS- Integrated Services; the congestion control mechanism should be different for different kinds of sources. Congestion control helps to provide priority differentiation of flows by servicing queues in different manners.

Indeed, the mechanisms to be considered in the data plane are:

- Traffic Scheduling: This provides service guarantees to time-critical applications. The scheduler first decides the order of requests to be served, and then it manages the queues of these awaiting requests. The scheduling can provide different services to the flows using parameters such as different bandwidths by serving only a single flow at a particular interval.

- Traffic Shaping: The goal here is to regulate average traffic rate and reduce congestion. The traffic shaping is performed at the boundary nodes. These nodes have classifiers that mark the flows according to their service requirements.

- Traffic Classification: The classification mechanisms provide identifying the traffic streams using packet contents or context information by using class-map commands.

- Traffic marking: This can be done using the set command which enables a wide range of packet marking criteria.

- Traffic Policing: The policing can be achieved by determining a rate threshold for a traffic class and then dropping any traffic that exceeds this rate.

- Traffic Queuing: The queuing always follows the classification and the marking. The queuing technologies mainly reside in a router or Layer 3 switch. There are a number of queuing techniques to be investigated.

\section{CONCLUSION}

In this paper, we have presented a framework proposal that relates QoE to QoS. Based on user perception and experience, the proposed approach will prioritize traffic packets of demanded application. The main goal is to satisfy the users and improve QoE according to the new concept of highest level services. This will facilitate the deployment of provisioned future e-society services. In a subsequent next paper, we are going to present the detailed results of our real life application case study and give our recommendations to enhance the performance of ISP networks.

\section{REFERENCES}

[1] Brooks, P., \& Hestnes, B. (2010). User measures of quality of experience: Why being objective and quantitative is important. IEEE Network, Volume:24, Issue: 2, pp. 8-13.

[2] Cherif, W., Ksentini, A., Negru, D., \& Sidibe, M. (2011). A_PSQA: Efficient real-time video streaming QoE tool in a future media internet context. IEEE International Conference on Multimedia and Expo (ICME), pp. $1-6$.

[3] Hoßfeld, T., Varela, M., \& Timmerer, C. (2012). Challenges of QoE Management for Cloud Applications. Communications Magazine, IEEE 50 (4), 28-36. 
[4] Khorsandroo, S., Noor, R. M., \& Khorsandroo, S. (2012). A mobility framework to enhance quality of experience through quality of service. International Conference on Innovation Management and Technology Research (ICIMTR), pp. 630-634.

[5] Vogel, A., Kerherve, B., von Bochmann, G., \& Gecsei, J. (1995). Distributed multimedia and QOS: a survey. Journal of Multimedia, IEEE, Vol.2, Issue 2, pp. 10-19.

[6] Xiuzhong Chen, Chunfeng Wang, Dong Xuan, Zhongcheng LiYinghua Min, \& Wei Zhao. (2003). Survey on QoS management of

[7] VoIP Zapater, M., \& Bressan, G. (2007). A Proposed Approach for Quality of Experience Assurance of IPTV. First International Conference on the Digital Society (ICDS 2007), 2-6 January 2007.

[8] Liu, P., Evans, S. C., \& Weerakoon, I. (2007). Multimedia QoS through content aware triage: An integrated DiffServ framework.

[9] Moller, L., Fischer, P., Moraes, F., Indrusiak, L. S., \& Glesner, M. (2010). Improving QoS of multi-layer Networks-on-Chip with partial and dynamic reconfiguration of routers.

[10] Alvarez, A., Cabrero, S., Paneda, X. G., Garcia, R., Melendi, D., \& Orea, R. (2011). A flexible QoE framework for video streaming services.

[11] Haryadi, S. (2012). QoS Measurement Of Telephony Services In 3G Networks Using Aggregation Method, 111-114.

[12] Malhotra, J. and Priyanka, V. S. (2014). Survey of Issues and Challenges in the Mac Layer for QoS in WiMAX. International Conference on Advances in Engineering and Technology Research (ICAETR), pp. $1-5$

[13] Kim, H. J., Lee, K. H., \& Zhang, J. (2010). In-service feedback QoE framework. Third International Conference on Communication Theory, Reliability, and Quality of Service (CTRQ), pp. 135 - 138.

[14] Chen, S. G., \& Park, K. (1999). An architecture for noncooperative QoS provision in many-switch systems. Proc. IEEE INFOCOM '99, pp. 864-872.

[15] Wu, C. C., Chen, K. T., Chang, Y. C., \& Lei, C. L. (2013). Crowdsourcing multimedia QoE evaluation: A trusted framework. IEEE Transactions on Multimedia, 15 (5), 1121-1137.

[16] Žagar, D. (2002). Formal specification of QoS parameters by hierarchical tree structure. 9th International Conference on Electronics, Circuits and Systems, 1075 - 1078 vol.3.

[17] Schmid, S. (1999). QoS based Real-Time Audio Streaming in The Internet. Master Thesis at the Universit"at Ulm Fakult"at f ur Informatik.

[18] Dogo, E. M., Ahmed, A., \& Olaniyi, O. M. (2014). Cross-Layer Integration Approach for Improving QoS for IPv6 Based VOIP, International Journal of Engineering and Technology, 4(8), 499505 .

[19] Agarwal, A. (2000). Quality of Service (QoS) in the New Public Network Architecture, IEEE Canadian Review- Fall/Automne 2000, 22-25.
[20] Bharat Bhargava (2002). Guest Editorial: Quality of Service in Multimedia Networks. Journal of Multimedia Tools and Applications, 17 (2-3), pp. 151-156.

[21] Ocevcic, H., \& Zagar, D. (2005). Quality of Service Management in Multimedia Communications. EUROCON 2005 - The International Conference on "Computer as a Tool".

[22] Onali, T. (2008). Quality of service technologies for multimedia applications in next generation network. Ph.D. Thesis, University of Cagliari.

[23] Brooks, P., \& Hestnes, B. (2010). User measures of quality of experience: Why being objective and quantitative is important. IEEE Network (Volume:24, Issue: 2 ), pp. 8 - 13.

[24] Ai-min, Z., Xue-song, Q., \& Luo-ming, M. (2011). An OSE proposing based customer QoE improvement method in the converged network. 3rd International Conference on Computer Research and Development (ICCRD 2011), Vol.2, pp. 70-75.

[25] Mitra, K., Zaslavsky, a, \& Ahlund, C. (2015). Context-Aware QoE Modelling, Measurement and Prediction in Mobile Computing Systems. IEEE Transactions on Mobile Computing, 14 (5), 920-936.

[26] Nortel Networks (2003) Introduction to Quality of Service (QoS)"White Paper", 1st edn., United States \& Canada: Nortel Networks.

[27] Laghari, K. R., Issa, O., Speranza, F., \& Falk, T. H. (2012). Quality-of-Experience Perception for Video Streaming Services : Preliminary Subjective and Objective Results. Signal \& Information Processing Association Annual Summit and Conference (APSIPA ASC), 2012 Asia-Pacific, pp. 1-9.

[28] Fiedler, M.; Hossfeld, T.; Tran-Gia, P.(2010). A generic quantitative relationship between quality of experience and quality of service. IEEE Network (Volume:24, Issue: 2 ), pp. 36 - 41.

[29] Kim, H.-J., Yun, D.-G., Kim, H.-S., Cho, K.-S., \& Choi, S.-G. (2012). QoE assessment model for video streaming service using QoS parameters in wired-wireless network. 14th International Conference on Advanced Communication Technology (ICACT), pp. 459 - 464.

[30] Delli Priscoli, F., Suraci, V., \& Pietrabissa, A. (2012). Modeling quality of experience in future internet networks. Future Network \& Mobile Summit 2012, Berlin, pp. 1-9,

[31] Stankiewicz, R., Cholda, P., \& Jajszczyk, A. (2011). QoX: What is it really?. IEEE Communications Magazine, 49 (4), pp. 148158.

[32] Skorin-Kapov, L., \& Varela, M. (2012). A multi-dimensional view of QoE: the ARCU model. Proceedings of the 35th International Convention, MIPRO, pp. 662-666.

[33] Khorsandroo, S., Noor, R. M., Khorsandroo, S. (2012). Stimuluscentric versus perception-centric relations in quality of experience assessment. Wireless Telecommunications Symposium (WTS), 2012, pp. 1-6.

[34] Malik, A., Qadir, J., Ahmad, B., Alvin Yau, K. L., \& Ubaid Ullah (2015). QoS in IEEE 802.11-based wireless networks: A contemporary review. Journal of Network and Computer Applications, Vol. 55, pp. 24-46. 